\title{
Internationale Zielmarktanalyse
}

\subsection{Segmentierung und Auswahl einer Weltmarktregion}

Abb. 3.1 zeigt die Auswertungsergebnisse von Baustein 1 nach Unternehmensgröße. Bei der Auswertung der Interviews konnten 14 Aussagen von Managern von Großunternehmen ISA-Modell Baustein 1 zugeordnet werden, die im Nachfolgenden ausführlich erläutert werden.

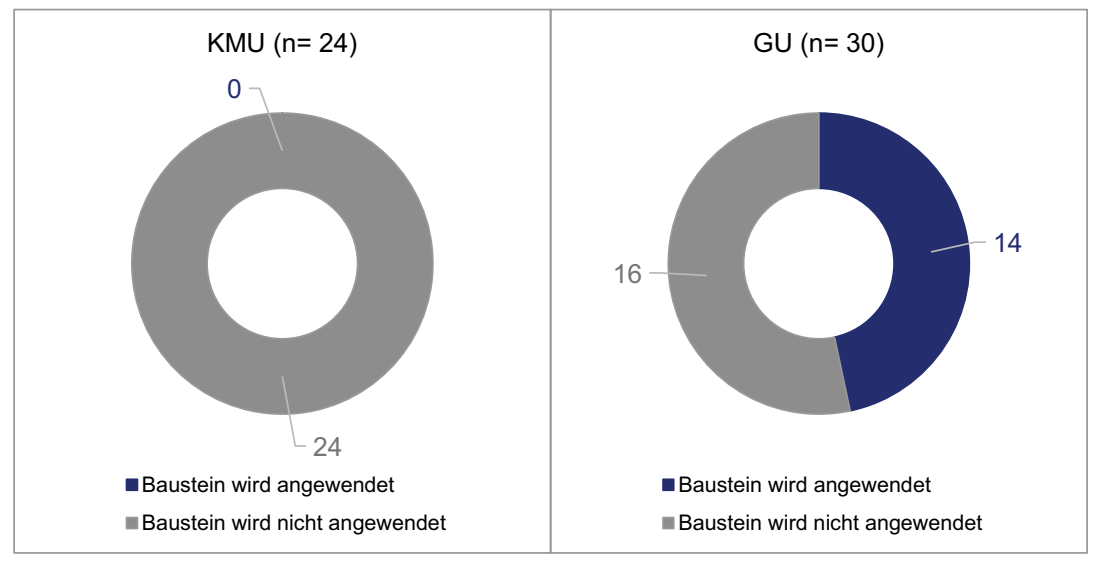

Abb. 3.1 Auswertungsergebnisse der Interviews zum ISA-Modell Baustein 1 nach Unternehmensgröße. (Quelle: Eigene Darstellung)

In Baustein 1 geht es um die Segmentierung und Auswahl einer Weltmarktregion. Deshalb wurde zuerst eine Auswertung der Segmentierungskriterien 
von Unternehmen durchgeführt. Die Ergebnisse können in Tab. 3.1 eingesehen werden. Viele Unternehmen wählen eine geographische Segmentierung der Weltregionen, um ihre Geschäfte international aufzustellen ${ }^{1}$. Ein weiteres Segmentierungskriterium ist eine volkswirtschaftliche Einordnung der Weltregionen. Während eines Interviews erklärte z. B. ein chinesischer Manager, dass sein Umwelttechnikunternehmen zunächst in Entwicklungsregionen expandieren wird, bevor entwickelte Regionen angegangen werden ${ }^{2}$. Weitere potenzielle Segmentierungskriterien sind eine zeitliche und eine industrielle Einordnung. Bei einer zeitlichen Einordnung z. B. spielen die Börsenzeiten in den Triade-Regionen Asien-Pazifik, Europa-Arabien-Afrika und Nord-\& Südamerika eine Rolle ${ }^{3}$. Eine industrielle Einordnung verfolgen z. B. länderübergreifende Cluster ${ }^{4}$.

Tab. 3.1 Unterschiedliche Segmentierungskriterien für Weltregionen

\begin{tabular}{l|l}
\hline Weltregionen & Segmentierungskriterium \\
\hline - Afrika & Geographische Segmentierung \\
- Amerika & \\
- Asien & \\
- Europa & \\
- Ozeanien & Volkswirtschaftliche Segmentierung \\
\hline - Entwickelte Regionen & \\
- Entwicklungsregionen & Zeitliche Segmentierung \\
\hline - Asien-Pazifik (APAC) & \\
- Europa-Arabien-Afrika (EMEA) & \\
- Nord- und Südamerika (AMER) & Industrielle Segmentierung \\
\hline - IT-Cluster in Silicon Valley & \\
- IT-Cluster in Shenzhen &
\end{tabular}

Quelle: Eigene Darstellung.

Nach der Auswertung der Interviews zur Segmentierung wurden die Tätigkeiten zur Auswahl der Weltmarktregionen analysiert. Die Tätigkeiten zur Auswahl der Weltmarktregion entsprechen dabei der externen Analyse des Prozesses

\footnotetext{
${ }^{1}$ Vgl. z. B. Interview 12015 oder Interview 392017.

${ }^{2} \mathrm{Vgl}$. Interview 152016.

${ }^{3}$ Vgl. Interview 192016.

${ }^{4}$ Vgl. Interview 112015 oder Interview 332017.
} 
des strategischen Managements, d. h. es werden Faktoren außerhalb des eigenen Unternehmens analysiert ${ }^{5}$. Die identifizierten Tätigkeiten können Abb. 3.2 entnommen werden.

Insbesondere bei den interviewten Umwelttechnikunternehmen spielen Umweltgesetze eine besondere Rolle bei der Entwicklung von Weltmärkten. So berichtet z. B. ein Manager, dass die Business Development Abteilung seines Unternehmens ein neues Gesetz der Europäischen Union für die Schwerölverbrennung bei der Binnenschifffahrt identifiziert hat. Sie entdeckten, dass die Produkte des Unternehmens bei der Einhaltung des neuen Gesetzes behilflich sein würden. Durch die externe Analysearbeit konnte das Vertriebspersonal aktiv auf die neuen Kunden zugehen. ${ }^{6}$ Andere Manager berichten, dass sie sehr genau regelmäBig und weltweit analysieren, wo z. B. die Technische Anleitung zur Reinhaltung der Luft (umgangssprachlich auch TA-Luft) vom Bundesministerium für Umwelt, Naturschutz und nukleare Sicherheit verabschiedet wird ${ }^{7}$. Zum Beispiel wurde die TA-Luft fast Eins zu Eins in Europa oder in bestimmten asiatischen oder lateinamerikanischen Ländern übernommen. Wichtig bei der Analyse der Gesetzeslage ist dabei nicht nur auf die Verabschiedung der entsprechenden Gesetze zu achten, sondern auch darauf, dass es prüfende Institutionen, wie z. B. den TÜV, gibt. $^{8}$

Ein weiteres wichtiges Kriterium zur Auswahl von Weltmarktregionen ist das Marktpotenzial bzw. das Marktwachstumspotenzial. Die Kennzahl Marktpotenzial wird dabei je nach Industrie unterschiedlich und teilweise mit Hilfe von Stellvertreter-Kennzahlen, wie z. B. Bevölkerungsentwicklung und Veränderung des Bruttoinlandsproduktes gemessen ${ }^{9}$. Außerdem gibt es zahlreiche Marktforschungsunternehmen, die sich auf die Ermittlung von Marktpotenzial in unterschiedlichen Industrien spezialisiert haben ${ }^{10}$. Dieses Wissen sollten Unternehmen unbedingt in ihre externe Analyse miteinbeziehen.

Weiterhin lässt sich aus Abb. 3.2 ablesen, dass ein nicht geringer Anteil der befragten Manager angibt, regelmäßig und weltweit Weltmarktregionen nach Trends in der Branche abzuscannen. In der Wasserwirtschaft, zum Beispiel, gibt es aktuell drei wichtige Trends: Die Automatisierung von Kläranlagen, die Einführung einer vierten Klärstufe zu Beseitigung von Hormonen im Abwasser und die

\footnotetext{
${ }^{5}$ Vgl. Hungenberg 2014, S. 9 ff.

${ }^{6} \mathrm{Vgl}$. Interview 12015.

${ }^{7} \mathrm{Vgl}$. Interview 4 2015, Interview 10 2015, Interview 162016.

${ }^{8}$ Vgl. Interview 42015.

${ }^{9}$ Vgl. z. B. Interview 12015.

${ }^{10}$ Vgl. Interview 1 2015, Interview 14 2016, Interview 39 2017, Interview 472018 und Interview 532017.
} 


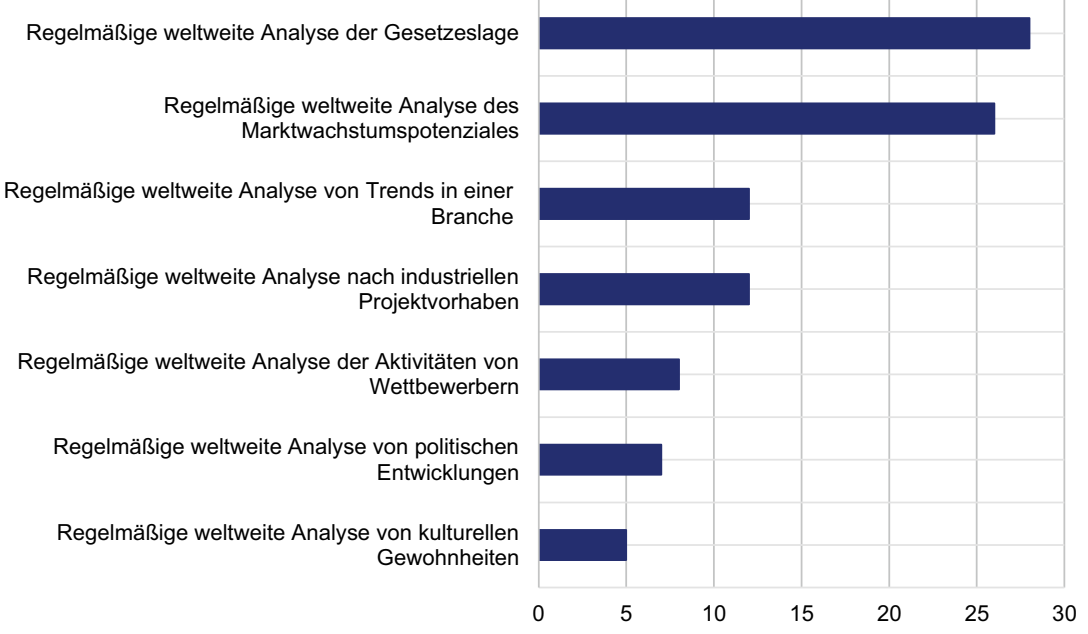

- Mehrfachnennung pro Interview möglich, wenn über mehrere Markteintritte gesprochen wurde.

Abb. 3.2 Auswertungsergebnisse der Interviews zum ISA-Modell Baustein 1 nach Tätigkeiten. (Quelle: Eigene Darstellung)

Weiterentwicklung von sogenannten Remote Monitoring Informationssystemen zur Überwachung der Wasserqualität in schwer zugänglichen Regionen ${ }^{11}$. Weitere Trends, die in den Interviews angesprochen wurden, sind z. B. Elektromobilität und Urbanisierung ${ }^{12}$. Es kann auch sinnvoll sein, sich laufend über Modernisierungswellen oder auslaufende Langzeitverträge mit Kommunen unterrichten zu lassen. In der Wasserwirtschaft, zum Beispiel, wird eine Modernisierungswelle in den nächsten 10 Jahren erwartet ${ }^{13}$. In der Abfall- und Kreislaufwirtschaft kann es hilfreich sein, die Verträge der Kommunen mit lokalen Unternehmen zu analysieren. Durch die europäische Union dürfen sich auch Unternehmen aus anderen europäischen Ländern auf lokale Ausschreibungen bewerben. ${ }^{14}$

Andere Unternehmen analysieren die Weltregionen nach industriellen Projektvorhaben. Dies können Vorhaben von bereits existierenden Kunden sein oder von potenziellen Neukunden. So erklärt z. B. ein Manager, dass er sich wöchentlich

\footnotetext{
${ }^{11}$ Vgl. Interview 112015.

${ }^{12}$ Vgl. Interview 82015 und Interview 512017.

${ }^{13} \mathrm{Vgl}$. Interview 112015.

${ }^{14}$ Vgl. Interview 132015.
} 
über industrielle Projektvorhaben von bereits existierenden Kunden informieren lässt ${ }^{15}$. Andere Manager legen wiederum den Fokus auf industrielle Projektvorhaben von potenziellen Neukunden ${ }^{16}$. Hierzu berichtet ein Manager, dass das Unternehmen regelmäßig die Weltmarktregionen nach geplanten neuen Fabriken analysiert. Anschließend wird eine Bewerbung beim potenziellen Kunden abgegeben, um möglichst früh an dem Projekt teilhaben zu können. ${ }^{17}$

Ein weiteres Element in Baustein 1 ist die regelmäßige und weltweite Analyse der Aktivitäten von Wettbewerbern und politischen Entwicklungen. Bei einem Unternehmen hat z. B. die Business Development Abteilung herausgefunden, dass es in dem betrachteten Land keinen Wettbewerber für eine bestimmte Technologie gibt $^{18}$. Andere beobachten politische Entwicklungen, wie z. B. die potenzielle Abschaffung der Sanktionen gegen den Iran, um als Erster im Markt zu sein, falls die Sanktionen fallen ${ }^{19}$.

Manche der interviewten Manager gaben an, in regelmäßigen Abständen weltweit nach Ressourcenvorkommen zu suchen. Zum Beispiel ist für viele Unternehmen aus der Luftreinhaltungsbranche der Bergbau oder die Ölindustrie eine wichtige Zielbranche. Überall dort wo neue Ressourcen entdeckt und abgebaut werden, gilt es wichtige Zielkunden zu erschließen. ${ }^{20}$

Weitere Faktoren, die bei der Auswahl von Zielmärkten von Bedeutung sind, sind z. B. kulturelle Gewohnheiten. Insbesondere in der Wasserwirtschaft, berichtet einer der Geschäftsführer, müssen bei Projekten in afrikanischen Ländern aufgrund der großen kulturellen Distanz, kulturelle Gewohnheiten vor Projektstart in Erfahrung gebracht werden. Zum Beispiel ist in vielen afrikanischen Ländern die Zahlungsmoral für Wasser selbst bei öffentlichen Einrichtungen nicht immer gegeben. Der Geschäftsführer erklärt, dass dies daran liegt, dass das Geld für das Wasser sowieso aus einem Topf kommt, weshalb sollte dann eine öffentliche Einrichtung, wie eine Behörde, ihren Wasserverbrauch bezahlen? ${ }^{21}$ Weitere kulturelle Gewohnheiten können das Verbraucherverhalten betreffen ${ }^{22}$ oder das Handelsverhalten ${ }^{23}$.

\footnotetext{
${ }^{15} \mathrm{Vgl}$. Interview 192016.

${ }^{16}$ Vgl. Interview 12015 und Interview 392017.

${ }^{17} \mathrm{Vgl}$. Interview 12015.

${ }^{18} \mathrm{Vgl}$. Interview 12015.

${ }^{19} \mathrm{Vgl}$. Interview 192016.

${ }^{20}$ Vgl. Interview 1 2015, Interview 8 2015, Interview 19 2016, Interview 572017.

${ }^{21} \mathrm{Vgl}$. Interview 52015.

${ }^{22}$ Vgl. Interview 82015 und Interview 352017.

${ }^{23}$ Vgl. Interview 132015.
} 
Lessons Learned in Baustein 1

Insgesamt konnte keine Aussage der KMUs diesem Baustein zugeordnet werden. Großunternehmen haben die nötigen Kapazitäten und Ressourcen eine regelmäBige und weltweite externe Analyse durchzuführen. Um konstant erfolgreich zu bleiben bzw. zu wachsen, sollten Unternehmen ihr Internationalisierungsmuster aber nicht mit den Bausteinen 2, 3 oder 4 des ISA-Modells beginnen, sondern mit den in Baustein 1 aufgezeigten Tätigkeiten kontinuierlich weltweite Informationen einholen. Wenn dies geschieht, kann zu einem bestimmten Zeitpunkt eine erfolgreiche Entscheidung zur Auswahl eines bestimmten Zielmarktes getroffen werden.

\subsection{Auswahl eines bestimmten Landes als prioritären Zielmarkt}

Aus Abb. 3.3 lassen sich die Auswertungsergebnisse zum ISA-Modell Baustein 2 nach Unternehmensgröße ablesen. Fünf Manager von KMUs gaben an, dass in ihrem Unternehmen ein neuer Zielmarkt zufällig durch einen Handelspartner auf internationalen Messen (siehe Baustein 3 des ISA-Modells) ausgewählt wurde ${ }^{24}$. Alle anderen 49 Manager gaben an, Zielmärkte aufgrund unterschiedlichster interner Analysen auszuwählen. Diese können in Abb. 3.4 eingesehen werden. Die Tätigkeiten zur Auswahl eines bestimmten Landes als prioritären Zielmarkt entspricht dabei der internen Analyse des Prozesses des strategischen Managements, d. h. es werden Faktoren innerhalb des eigenen Unternehmens analysiert ${ }^{25}$.

Diverse Manager gaben an, dass sie neue Zielmärkte aufgrund von Ressourcenoptionen ausgewählt haben. Zum Beispiel berichtet einer der Geschäftsführer, dass ein neuer Ländermarkt erschlossen wurde, weil ein langjähriger Mitarbeiter aus persönlichen Gründen in diesen Ländermarkt umziehen musste ${ }^{26}$. Ein anderer Geschäftsführer entschied sich für den Markteintritt in den chinesischen Markt aufgrund günstiger humaner Ressourcen ${ }^{27}$.

Eine weitere Möglichkeit neue Zielmärkte auszuwählen ist die Auslandsaktivitäten von Großkunden zu verfolgen. Mehrere Manager gaben an, dass die

\footnotetext{
${ }^{24}$ Vgl. Interview 38 2017, Interview 61 2017, Interview 67 2017, Interview 732017 und Interview 792018.

${ }^{25}$ Vgl. Hungenberg 2014, S. 9 ff.

${ }^{26} \mathrm{Vgl}$. Interview 32015.

${ }^{27}$ Vgl. Interview 422017.
} 


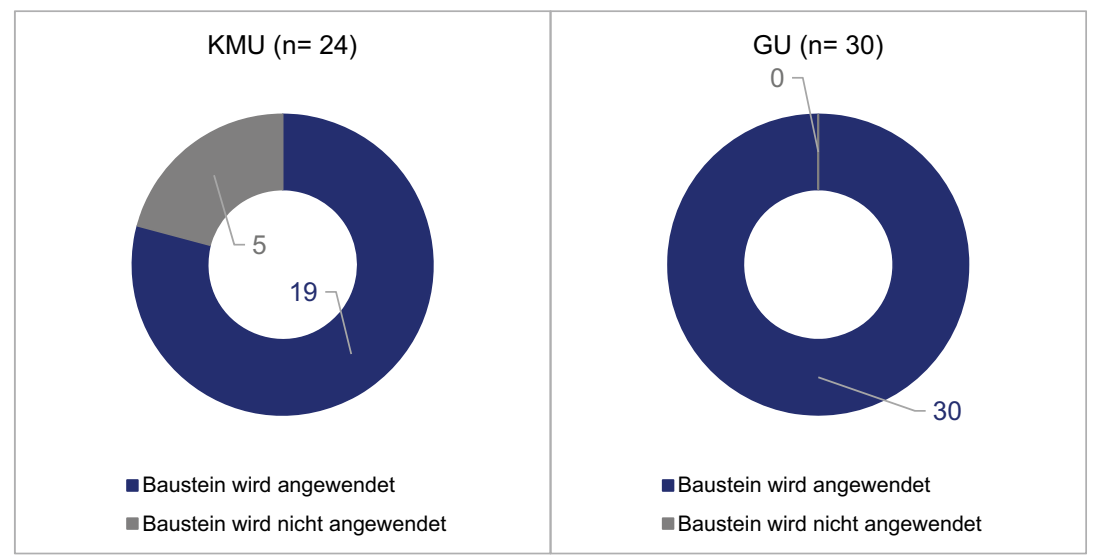

Abb. 3.3 Auswertungsergebnisse der Interviews zum ISA-Modell Baustein 2 nach Unternehmensgröße. (Quelle: Eigene Darstellung)

Analyse von Ressourcen

Analyse der Auslandsaktivitäten von Großkunden

Analyse von langfristigen potentiellen Märkten

Analyse der vorhandenen Geschäftskontakten

Analyse von Einflussnahmemöglichkeiten auf Stakeholder

Erstellen eines eigenen Auswahlmodells

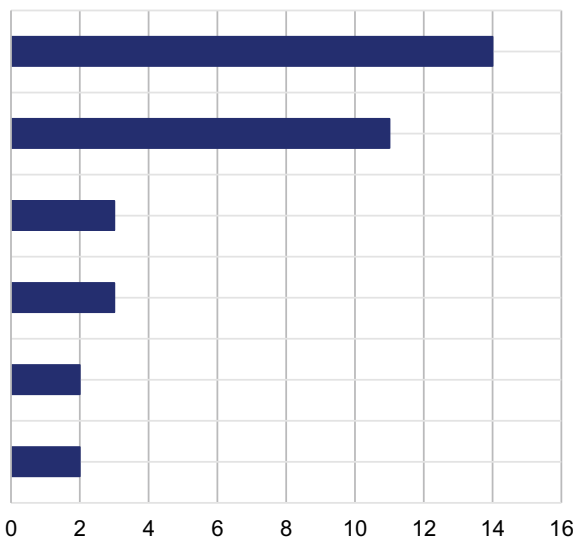

- Mehrfachnennung pro Interview möglich, wenn über mehrere Markteintritte gesprochen wurde.

Abb. 3.4 Auswertungsergebnisse der Interviews zum ISA-Modell Baustein 2 nach Tätigkeiten. (Quelle: Eigene Darstellung) 
Zielmarktauswahl feststand als einer ihrer Großkunden beschloss in den Auslandsmarkt einzutreten ${ }^{28}$. Viele Umwelttechnikunternehmen haben z. B. deutsche Automobilhersteller als Großkunden ${ }^{29}$. Wenn diese beschließen in den chinesischen Markt einzutreten, bleibt vielen Zulieferern keine andere Wahl als ebenfalls in den Markt einzutreten.

Eine weitere Tätigkeit in ISA-Modell Baustein 2 ist die Analyse von langfristigen potenziellen Märkten ${ }^{30}$. Einer der Geschäftsführer definiert in regelmäßigen Abständen langfristig strategisch relevante Märkte für seine Industrie. Für das Unternehmen waren z. B. in der Vergangenheit China, Russland und SaudiArabien strategisch wichtige Märkte, da in diesen Märkten viel gebaut wurde und immer noch viel gebaut wird. In diesen Märkten werden oft Trends gesetzt und eine Absenz würde in dieser Industrie z. B. bedeuten, dass andere Zertifikatssysteme durchgesetzt werden, bei denen das Unternehmen dann keinen Einfluss mehr nehmen kann. Die Folge wäre eine Anpassung der eigenen Bauweise, die wiederum mit Kosten verbunden ist. ${ }^{31}$

Mehrere Manager von KMUs berichten, dass sie einen Geschäftskontakt in einem Markt hatten und dass sie deshalb in den Markt eingetreten sind ${ }^{32}$. Einzelne Unternehmen betreiben aber auch Aufklärungsarbeit in eigener Sache. Zum Beispiel ist der technisch mögliche Stand vielen Kommunen oft nicht bewusst. In diesem Fall sprechen die Unternehmen gezielt wichtige Stakeholder an. ${ }^{33}$

Zwei der befragten Manager gaben an, dass sie für ihr Unternehmen ein eigenes Marktauswahlmodell entworfen haben ${ }^{34}$. Das erste Unternehmen ist in der Abfall- und Kreislaufwirtschaft tätig. Der Geschäftsführer berichtet, dass die Zielmärkte nach den unterschiedlichsten Kriterien bewertet wurden, welche teilweise aus Projekttreibern und Projekthemmnissen abgeleitet wurden. Kriterien für das sogenannte Abfallmarktmodell waren z. B. Abfallaufkommen, Deponiequote, Verträge mit Kommunen, politische Verhältnisse, Wahrscheinlichkeit von Bürgerinitiativen, industrielles Umfeld, Netzanschlüsse, Brennstoffnutzung, juristisches System, Korruptions-Index, kulturelle Faktoren und Umfeld für Lieferanten $^{35}$. Nach der Sammlung der wichtigsten Kriterien wurde für jeden Zielmarkt

${ }^{28} \mathrm{Vgl}$. z. B. Interview 72015 oder Interview 102015.

${ }^{29} \mathrm{Vgl}$. z. B. Interview 12015 oder Interview 162016.

${ }^{30} \mathrm{Vgl}$. Interview 1 2015, Interview 12, 2015, Interview 162016.

${ }^{31} \mathrm{Vgl}$. Interview 122015.

${ }^{32}$ Vgl. z. B. Interview 242016 und Interview 292016.

${ }^{33} \mathrm{Vgl}$. Interview 11 2015, Interview 162016.

${ }^{34}$ Vgl. Interview 132015 und Interview 472018.

${ }^{35}$ Die aufgeführte Auswahl an Kriterien ist nicht abschließend aufgeführt. 
im Abfallmarktmodell ein Ländersteckbrief erstellt. Die Kriterien wurden z. B. mit Hilfe von EU-Statistiken etc. gefüllt. ${ }^{36}$ Das zweite Unternehmen ist in der Lebensmittelindustrie tätig. Der International Sales Manager erläutert, dass sein internationales Marktauswahlmodell zwei wichtige Kennzahlen beinhaltet: Marktpotenzial und Marktbewertung. Die Kennzahlen für das Marktpotenzial werden mit Daten und Fakten aus objektiven Studien gefüttert. Die Marktbewertung ist eine subjektive Einschätzung des Managers. Der Manager berichtet zudem, dass es ausreichend ist, wenn ein Länderscoringmodell einmal erstellt wird. In den letzten 10 Jahren hat sich die Länderreihenfolge in seiner Industrie z. B. nicht verändert. $^{37}$

\section{Lessons Learned in Baustein 2}

Baustein 2 wird sowohl von KMUs als auch von Großunternehmen angewendet. Bei ein paar wenigen KMUs konnte keine Aussage Baustein 2 zugeordnet werden. Diese KMUs überspringen die Bausteine 1, 2 und ggfs. auch 3 (siehe Tab. 5.1 in Abschnitt 5.1). Die Auswertung der Interviews zu Baustein 2 zeigt, dass es Unternehmen leichter fällt Markteintrittspunkte zu sammeln, wenn sie regelmäBig eine interne Analyse zu den Zielmärkten unternehmen. Außerdem ist es hilfreich ein eigenes Marktauswahlmodell zu Beginn eines eigenen Internationalisierungsmusters aufzustellen. Anschließend kann das eigene Marktauswahlmodell in regelmäßigen Abständen aktualisiert werden und mit der Sammlung von ersten Markteintrittspunkten z. B. zu den Top 3 Zielmärkten begonnen werden.

\subsection{Sammlung von Markteintrittspunkten im Zielmarkt}

In Abb. 3.5 sind die Auswertungsergebnisse zum ISA-Modell Baustein 3 nach Unternehmensgröße dargestellt. Abb. 3.5 zeigt, dass fast alle interviewten Unternehmen Markteintrittspunkte im Zielmarkt sammeln ${ }^{38}$. Zwei Aussagen von KMUs zeigten, dass sie direkt eine Markteintrittsstrategie gewählt haben, weil die Zielmärkte zu wichtig waren, als dass sie nicht vor Ort eine eigene Vertriebsniederlassung besitzen.

Wie die Tätigkeiten zu diesem Baustein aussehen können, kann aus Abb. 3.6 abgelesen werden. Messen sind mit großem Abstand die beliebtesten Markteintrittspunkte. Messen wurden über alle Industrien und Unternehmensgrößen hinweg von besonderer Relevanz beim Aufbau von Geschäftsbeziehungen in

\footnotetext{
${ }^{36} \mathrm{Vgl}$. Interview 132015.

${ }^{37} \mathrm{Vgl}$. Interview 472018.

${ }^{38}$ Vgl. Ausnahmen siehe Interview 732017 und Interview 792018.
} 


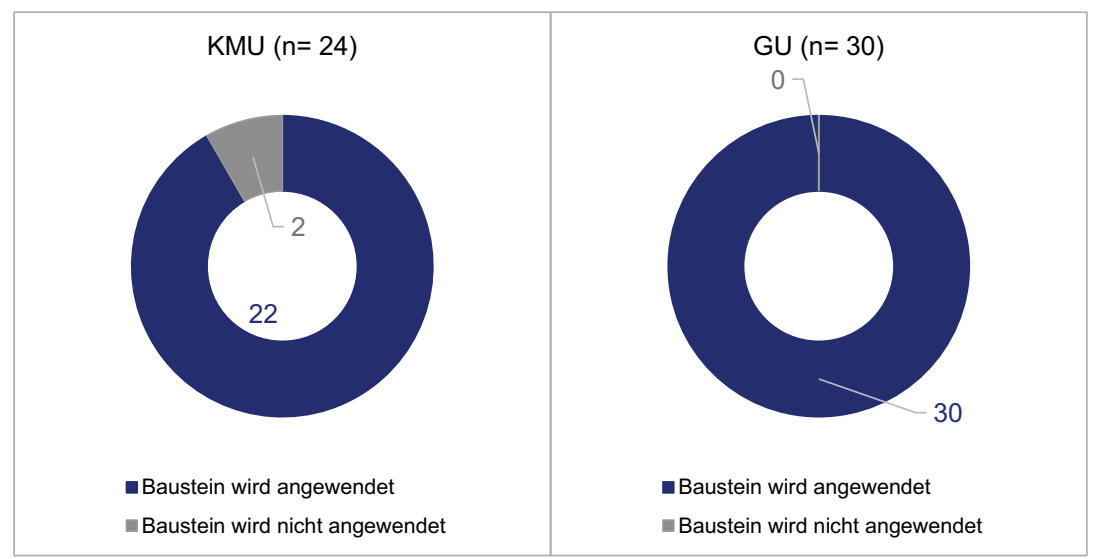

Abb. 3.5 Auswertungsergebnisse der Interviews zum ISA-Modell Baustein 3 nach Unternehmensgröße. (Quelle: Eigene Darstellung)

ausländischen Märkten erwähnt ${ }^{39}$. In der Umwelttechnikindustrie, z. B. ist eine der wichtigsten Messen die IFAT. Sie findet jedes zweite Jahr in München statt und ist die Weltleitmesse für Wasser-, Abwasser, Abfall- und Rohstoffwirtschaft. Außerdem gibt es Ableger von der Messe in Südafrika, Indien, China und in der Türkei. ${ }^{40}$ Darüber hinaus besuchte ein Interviewpartner von einem Umwelttechnikunternehmen die Leitmessen der Kunden z. B. aus der Chemieund Pharmaindustrie, um dort die Kunden auf das Unternehmen aufmerksam zu machen ${ }^{41}$. Ein anderer Interviewpartner erklärt, dass das Management nach einem Markteintritt in den chinesischen Markt viel Zeit darauf verwendet hat, geeignete Messen zu identifizieren und auch gegebenenfalls im Nachhinein wieder Messen aussortiert hat, da diese z. B. in kleinen Turnhallen stattfanden ${ }^{42}$. Viele der interviewten Manager berichten, dass sie über Messen erste Handelspartner in den ausländischen Märkten gefunden haben und so der Markteintritt letztendlich in das Land stattgefunden hat $^{43}$.

\footnotetext{
${ }^{39}$ Vgl. z. B. Interview 24 2016, Interview 1 2015, Interview 42015.

${ }^{40}$ Vgl. Messe München 2018.

${ }^{41} \mathrm{Vgl}$. Interview 12015.

${ }^{42} \mathrm{Vgl}$. Interview 392017.

${ }^{43}$ Vgl. z. B. Interview 7 2015, Interview 8 2015, Interview 382017 oder Interview 46, um nur einige Beispiele zu nennen.
} 


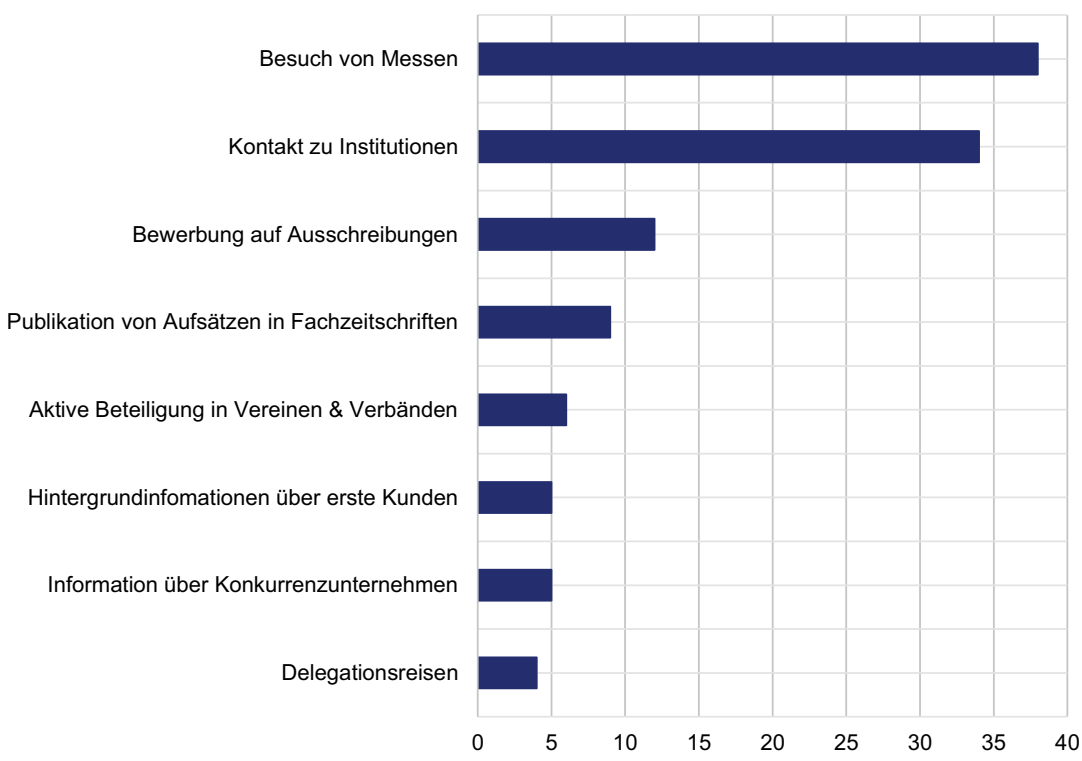

- Mehrfachnennung pro Interview möglich, wenn über mehrere Markteintritte gesprochen wurde.

Abb. 3.6 Auswertungsergebnisse der Interviews zum ISA-Modell Baustein 3 nach Tätigkeiten. (Quelle: Eigene Darstellung)

Nach den Messebesuchen wurden Kontakte zu Institutionen wie die Industrieund Handelskammer, die Außenhandelskammern (im Nachfolgenden bezeichnet als AHKs), Germany Trade \& Invest, Universitäten ${ }^{44}$, Institute, Marktforschungsunternehmen, Beratungsunternehmen, Spezialbörsen, Entwicklungsbanken, Wirtschaftsförderungsagenturen und Regierungen als beliebte Markteintrittspunkte angegeben $^{45}$. Wie z. B. die AHKs Unternehmen bei der Sammlung von Markteintrittspunkten helfen können, wird in den nachfolgenden Abschnitten anhand der AHK Mexiko, der Europäischen Handelskammer in China, der AHK China und der AHK USA ausführlich erläutert.

AHK Mexiko: Die AHK in Mexiko-Stadt bietet z. B. unternehmensbezogene Marktstudien, Standortanalysen sowie Ursprungsanalysen an. Darüber hinaus werden auch Adressrecherchen, Firmenauskünfte sowie Geschäftspartnersuchen

\footnotetext{
${ }^{44} \mathrm{Vgl}$. Interview 582017 und Interview 622017.

${ }^{45}$ Vgl. z. B. Interview 2 2015, Interview 3 2015, Interview 5 2015, Interview 142016 , Interview 35 2017, Interview 36 2017, Interview 38 2017, Interview 47 2018, Interview 512017.
} 
angeboten. ${ }^{46}$ Im Nachfolgenden werden einzelnen Dienstleistungen, die für den Markteintrittspunkt von Relevanz sind, näher erläutert:

Marktstudien: Bei den Marktstudien gilt es dem Unternehmen unter anderem produktspezifische Import- und Exportstatistiken zu erstellen sowie eine Übersicht über Importbestimmungen zu geben. Grundlage der Import- und Exportstatistiken sind die Zolltarifnummern der jeweiligen Länder, welche auf dem sechsstelligen HS-Code aufbauen. Der sechsstellige HS-Code ist für alle Mitglieder der Weltzollorganisation identisch und bietet eine Grundlage für eine einheitliche Warenklassifizierung (siehe auch Fallstudie 6.1). Die Interviewpartnerin erklärt, dass sie für ihre Arbeit sowohl die zwölfstelligen europäischen als auch die achtstelligen mexikanischen Zolltarifnummern benötigt. Über die Daten des mexikanischen Wirtschaftsministeriums (Secretaría de Economía) und deren Sistema de Información Arancelaria via Internet (Im Nachfolgenden bezeichnet als SIAVI) kann sie für deutsche Unternehmen die den deutschen/europäischen Zolltarifnummern entsprechenden mexikanischen Warennummer identifizieren und die entsprechenden mexikanischen Import- und Exportstatistiken recherchieren. Die einzelnen produktspezifischen Importbestimmungen können ebenfalls über SIAVI und andere Datenbanken herausgefunden werden. Außerdem müssen bei der Analyse die anfallenden nicht-tarifären Importbestimmungen wie Importverbote - z. B. von deutschem Schweinefleisch -, Importgenehmigungen, zu erfüllende mexikanische offizielle Normen, usw. beachtet werden. ${ }^{47}$

Standortanalyse: Bei Standortanalysen werden zunächst die Anforderungen eines Unternehmens an den neuen Produktionsstandort und dessen Umgebung abgefragt und je nach dem zu beliefernden Markt einige Bundesstaaten vorgeschlagen. Anschließend überprüfen die Mitarbeiter der AHK Mexiko diese Bundesstaaten und deren Industrieparks, welche derselben den Anforderungen des Unternehmens genügen, um dann dem deutschen Investor einen Bericht mit einer detaillierten Darstellung der verschiedenen Optionen unterbreiten zu können. Als allgemeine Informationen zu den diversen Bundesstaaten dient z. B. die Webseite von ProMexiko. Die Standortanalyse wird nur bei Unternehmen durchgeführt, die eine Produktion in Mexiko planen. ${ }^{48}$

Ursprungsanalyse: Insgesamt hat Mexiko mit 46 Ländern Handelsabkommen abgeschlossen. In der Regel haben Produkte, die einen Anteil von 65 Prozent Wertschöpfung in Mexiko haben und damit den mexikanischen Ursprung erhalten, im Rahmen dieser Abkommen niedrigere oder keine Importzölle. Wenn ein

\footnotetext{
${ }^{46}$ Vgl. AHK Mexiko 2018.

${ }^{47} \mathrm{Vgl}$. Interview 322016.

${ }^{48}$ Vgl. Interview 322016.
} 
Unternehmen z. B. Solarzellen mit Ursprung in Deutschland nach Mexiko importiert, und in Mexiko nur ein Rahmen um die Solarzellen gebaut wird, dann reicht dieser Wertschöpfungsanteil nicht aus, um die Solarmodule z. B. von Mexiko aus in die USA zu importieren. Um im Zielland geringe Importzölle zu bezahlen, muss das Unternehmen mehr von seiner Wertschöpfung der Solarzellen in Mexiko produzieren lassen. Die Ursprungsanalyse der Interviewpartnerin ist sehr aufwändig und sie benötigt dazu von den Unternehmen viele Informationen, wie zum Beispiel, die Zolltarifnummern des Endproduktes und der Vorerzeugnisse, die Ursprungszeugnisse der Vorerzeugnisse und der Verarbeitungsstand des Produktes aus dem Land, aus dem es exportiert wird. Mit Hilfe der erhaltenen Informationen kann sie analysieren welche Bestimmungen im Rahmen der diversen Freihandelsabkommen für das vorliegende Produkt gelten. Wird z. B. ein Anteil von 65 Prozent der Wertschöpfung in Mexiko erreicht, kann ein europäisches Unternehmen die Ausstellung einer Warenverkehrsbescheinigung EUR.1 beim mexikanischen Wirtschaftsministerium beantragen. Mit der Warenverkehrsbescheinigung EUR.1 müssen Unternehmen nicht den vollen Zollsatz bezahlen. Im umgekehrten Fall wird diese Bescheinigung von Zollämtern erstellt. Unter www.sice.oas.org können sämtliche Freihandelsabkommen von Mexiko und darüber hinaus von allen nord- und südamerikanischen Ländern eingesehen werden. In den Freihandelsabkommen stehen die Wertschöpfungsanteile, die teilweise nach Industrien oder sogar spezifischen Produkten variieren. ${ }^{49}$

Weitere Institutionen die deutschen Unternehmen beim Markteintritt in Mexiko helfen sind z. B. die German Centre for Industry and Trade Mexico ${ }^{50}$ sowie die deutsche Gesellschaft für internationale Zusammenarbeit ${ }^{51}$. Die Standorte der German Centre sind allesamt in Schwellenländern und sollen den Markteintritt in diese Länder erleichtern. Der große Vorteil den Unternehmen durch die German Centre haben, ist, dass sie mit nur wenig Aufwand ein Büro anmieten und das Netzwerk vor Ort nutzen können. In Mexiko-Stadt sind derzeit 110 Mieterfirmen untergebracht, die alle dasselbe Ziel haben: den mexikanischen Markt zu erschließen. Um den Austausch untereinander zu fördern, findet zweimal pro Jahr das B-to-B-Netzwerktreffen statt. Unter den 110 Mieterfirmen befinden sich aber nicht nur kleine und mittlere deutsche Unternehmen, sondern auch z. B. mexikanische Anwälte, die Deutsch sprechen, sowie Banker von der LBBW, die die Eigentümerin der German Centre ist. ${ }^{52}$

\footnotetext{
${ }^{49} \mathrm{Vgl}$. Interview 322016.

${ }^{50} \mathrm{Vgl}$. Interview 232016.

${ }^{51} \mathrm{Vgl}$. Interview 302016.

${ }^{52} \mathrm{Vgl}$. Interview 232016.
} 


\section{Europäische Handelskammer in China:}

Bei der Festlegung eines Markteintrittspunktes im chinesischen Markt können deutsche Unternehmen sich ebenfalls an die AHK wenden sowie an Wirtschaftsförderungsgesellschaften. Darüber hinaus ist die europäische Handelskammer Ansprechpartner für europäische sowie chinesische Unternehmen vor Ort. Den Unterschied zwischen den Handelskammern macht folgendes Zitat eines Mitarbeiters der europäischen Handelskammer deutlich:

"[...] there is also no contradiction between the European Chamber and the national chambers, because ask the Frenchmen or Swiss or any other nation, it is easier to start [Anmerk. d. Verf.: in a foreign country] with your countrymen. The European Chamber of Commerce, we are here for the established companies that need help to lobbying / policing in China. We are complementing each other quite well." 53

Die europäischen Handelskammern setzen sich für die Durchsetzung europäischer Interessen ein und machen z. B. auf Missstände in den ausländischen Direktinvestitionsbedingungen aufmerksam ${ }^{54}$. Die lokalen und länderspezifischen Handelskammern werden von der europäischen Handelskammer als wichtige Markteintrittspunkte gesehen ${ }^{55}$.

\section{AHK China:}

Ein Mitarbeiter der AHK in Chengdu hat sich unter anderem auf die Markteintrittsstrategie des grenzüberschreitenden elektronischen Handels spezialisiert, welcher in China sehr im Trend liegt und im Vergleich zum stationären Handel Erleichterungen für ausländische Unternehmen verspricht (siehe Fallstudie 6.2.4) ${ }^{56}$. Weiterhin rät der Mitarbeiter deutschen Unternehmen sich Gedanken über die Kunden zu machen und auch mit chinesischen Kunden vorab zu reden. Darüber hinaus sollte eine eigene Präsenz mit 1-2 Mitarbeitern im chinesischen Markt aufgebaut werden. Bei der Standortwahl im chinesischen Markt geht es entweder nach Tradition, d. h. Shanghai, nach Sitz der Kunden oder Industriezugehörigkeit. ${ }^{57}$

\footnotetext{
${ }^{53}$ Interview 372017.

${ }^{54} \mathrm{Vgl}$. Interview 372017.

${ }^{55} \mathrm{Vgl}$. Interview 372017.

${ }^{56} \mathrm{Vgl}$. Interview 342017.

${ }^{57} \mathrm{Vgl}$. Interview 342017 und Interview 63.
} 


\section{U.S. und lateinamerikanische Handelskammern:}

In anderen Ländern gibt es ebenfalls AHKs, die sich um die Internationalisierung der eigenen Unternehmen kümmern. In den USA, z. B., gibt es neben den lokalen Handelskammern sowie den AHKs noch weitere Institutionen, die bei einer Internationalisierung helfen können. Diese sind die folgenden Institutionen: Foreign Agricultural Service, Department of Energy, Small Business Administration oder International Trade Administration ${ }^{58}$. Die International Trade Administration bietet z. B. Marktforschungsdienstleistungen, internationale Partnersuche sowie Exportberatungen an. Die Experten vom U.S. Commercial Service erstellen z. B. anhand der HS Codes der Weltzollorganisation für ein interessiertes Unternehmen Export- und Importstatistiken zusammen, anschließend entwickeln sie gemeinsam mit einem U.S. Unternehmen eine Markteintrittsstrategie für den jeweiligen Zielmarkt. Laut dem U.S. Commercial Service in Austin, Texas, ist für texanische Unternehmen Mexiko der Zielmarkt Nummer 1. ${ }^{59}$ Es gibt sogar eine gemeinsame Handelskammer der beiden Länder - die U.S.-Mexico Chamber of Commerce $^{60}$. Die U.S.-Mexico Chamber of Commerce ist zuständig für Unternehmen die Geschäfte im jeweiligen anderen Land tätigen wollen. Ein Mitarbeiter der U.S.-Mexico Chamber of Commerce erläutert, dass es für Unternehmen wichtig ist, die richtigen Quellen zu kennen, z. B. gibt es die National Chamber of Transforming Industries, die National Chamber of Commerce, Tourism and Services, das Instituto Nacional de Estadística y Geografía oder die Banca Nacional Financiera ${ }^{61}$. Weitere Wirtschaftsförderungsgesellschaften in Lateinamerika sind die Geschäftsentwicklungsagenturen der jeweiligen Länder z. B. ProMéxico, ProColumbia, ProHonduras, etc ${ }^{62}$.

Für viele der befragten Umwelttechnikunternehmen spielen zudem Ausschreibungen eine wichtige Rolle ${ }^{63}$. Ohne einen Zuschlag einer Ausschreibung könnten viele Unternehmen ihr Internationalisierungsmuster nicht oder nur in Zusammenarbeit mit anderen Unternehmen beginnen. Dabei kann eine Angebotsabgabe ein

\footnotetext{
${ }^{58} \mathrm{Vgl}$. Interview 212016.

${ }^{59} \mathrm{Vgl}$. Interview 212016.

${ }^{60} \mathrm{Vgl}$. Interview 262016.

${ }^{61} \mathrm{Vgl}$. Interview 262016.

${ }^{62} \mathrm{Vgl}$. Interview 212016.

${ }^{63} \mathrm{Vgl}$. Interview 2 2015, Interview 5 2015, Interview 7 2015, Interview 12 2015, Interview 13 2015, Interview 25 2016, Interview 27 2016, Interview 36 2017, Interview 41 2017, Interview 51 2017, Interview 532017 und Interview 662017.
} 
Unternehmen zwischen 2-3 Mio. EUR kosten und pro Ausschreibung mehrere tausend Seiten umfassen ${ }^{64}$.

Verschiedene Manager berichten zudem, dass es sinnvoll ist in Fachzeitschriften zu publizieren ${ }^{65}$. Wichtige Fachzeitschriften gibt es in jedem Land zu jeder Industrie. In Mexiko, zum Beispiel, ist die Zeitschrift Agua y Saneamiento des ANEAS-Wasserverbandes von großer Bedeutung ${ }^{66}$. Weitere wichtige Fachzeitschriften im Segment der Wasserwirtschaft sind z. B. die Zeitschriften Waste Water International, Global Water International and Process ${ }^{67}$.

\begin{abstract}
„Am besten ist dann eine Kombination von Anzeige, Editorial oder Referenzinformation in einer Zeitschrift. Gerade in der Umwelttechnikbranche in Deutschland läuft sehr viel über diese Zeitschriften. Man merkt sehr deutlich die Rückläufe, nachdem man einen Artikel veröffentlich hat. “68
\end{abstract}

Unternehmen sollten für ihre Industrie die wichtigste Fachzeitschrift identifizieren und dort Referenzprojekte publizieren.

Oftmals haben die einzelnen Industriezweige auch eigene Vereine und Verbände. Diese gilt es zu identifizieren ${ }^{69}$. In der Abfall- und Kreislaufwirtschaft z. B. gibt es den Bundesverband deutscher Entsorger oder die Bundesvereinigung Deutscher Stahlrecycling- und Entsorgungsunternehmen ${ }^{70}$. In der Wasserwirtschaft gibt es den Verein German Water Partnership ${ }^{71}$ oder den mexikanischen Verein ANEAS ${ }^{72}$.

Eine weitere wichtige Tätigkeit in Baustein 3 des ISA-Modells ist die Einholung von Informationen über potenzielle Kunden und Wettbewerber ${ }^{73}$. Das Sammeln von Hintergrundinformationen über die Kunden oder Wettbewerber kann den tatsächlichen Markteintrittspunkt bzw. die Markteintrittsregion beeinflussen (siehe auch Abschn. 5.1). Zum Beispiel wählt ein chinesisches Unternehmen nur Städte in

\footnotetext{
${ }^{64}$ Vgl. Interview 132015.

${ }^{65}$ Vgl. Interview 2 2015, Interview 16 2016, Interview 25 2016, Interview 29 2016, Interview 60 2017, Interview 67 2017, Interview 71 2017, Interview 772017 und Interview 78 2017.

${ }^{66} \mathrm{Vgl}$. Interview 292016.

${ }^{67} \mathrm{Vgl}$. Interview 162016.

${ }^{68}$ Interview 162016.

${ }^{69} \mathrm{Vgl}$. Interview 32015 , Interview 122015.

${ }^{70} \mathrm{Vgl}$. Interview 22015.

${ }^{71} \mathrm{Vgl}$. Interview 72015 und Interview 542017.

${ }^{72} \mathrm{Vgl}$. Interview 292016.

${ }^{73}$ Vgl. z. B. Interview 3 2015, Interview 142016 oder Interview 152016.
} 
anderen Schwellenändern oder Entwicklungsländern aus, wo die Kunden ein relativ hohes Einkommen haben und die Luftqualität schlecht ist ${ }^{74}$.

Eine Mitarbeiterin der AHK in Mexiko erklärt, dass auf Bundesebene z. B. das Bundesministerium für Wirtschaft und Energie Zielmarktanalysen bei den AHKs in Auftrag gibt, um zukünftige Delegationsreisen zu planen ${ }^{75}$. Dazu müssen die Mitarbeiterin und ihre Kollegen Informationen über die Wachstumsmärkte z. B. in Mexiko heraussuchen. Weiterhin erklärt sie:

\begin{abstract}
„Nach einer erfolgreichen Erstellung einer Zielmarktanalyse beginnt in der Regel der Auftraggeber in Deutschland Werbung für eine Delegationsreise zu machen. Oft müssen die Unternehmen erst in Deutschland akquiriert werden. Die AHK Mexiko organisiert ihrerseits mexikanische Unternehmen in dem jeweiligen Bereich. Nach erfolgreicher Akquisition in beiden Ländern, erfolgt eine Reise ins Zielland. Auf einer Fachkonferenz können die Unternehmen Vorträge halten, sich kennen lernen und vernetzen." $" 76$
\end{abstract}

Dabei sind die Gründe für eine Teilnahme bei einer Delegationsreise unterschiedlich: während der eine Interviewpartner auf Delegationsreisen geht um Handelspartner zu akquirieren ${ }^{77}$, nimmt der andere Interviewpartner teil, um den Bekanntheitsgrad der eigenen Marke sowohl im Inland wie im Ausland zu erhöhen ${ }^{78}$. Darüber hinaus bieten Delegationsreisen die Chance in Kontakt mit lokalen Politikern zu kommen, was insbesondere in Schwellenländern bei öffentlichen Aufträgen von großem Vorteil sein kann ${ }^{79}$.

Lessons Learned in Baustein 3

In Baustein 3 gibt es sehr viele Tätigkeiten, die einem Unternehmen helfen können Informationen über den Zielmarkt zu gewinnen. Einige der befragten Manager gaben an, dass sie in regelmäßigem Austausch mit den in Abb. 3.6 genannten Institutionen sind. Daher sollten Unternehmen an einem regelmäßigen Kommunikationsaustausch mit Institutionen im Zielmarkt arbeiten. Je mehr Informationen ein Unternehmen gewinnen kann, desto erfolgreicher kann es an einer Markteintrittsstrategie arbeiten.

\footnotetext{
${ }^{74} \mathrm{Vgl}$. Interview 562017.

${ }^{75} \mathrm{Vgl}$. Interview 222016.

${ }^{76}$ Interview 222016.

${ }^{77} \mathrm{Vgl}$. Interview 72015.

${ }^{78} \mathrm{Vgl}$. Interview 662017.

${ }^{79} \mathrm{Vgl}$. Interview 662017.
} 
Open Access Dieses Kapitel wird unter der Creative Commons Namensnennung 4.0 International Lizenz (http://creativecommons.org/licenses/by/4.0/deed.de) veröffentlicht, welche die Nutzung, Vervielfältigung, Bearbeitung, Verbreitung und Wiedergabe in jeglichem Medium und Format erlaubt, sofern Sie den/die ursprünglichen Autor(en) und die Quelle ordnungsgemäß nennen, einen Link zur Creative Commons Lizenz beifügen und angeben, ob Änderungen vorgenommen wurden.

Die in diesem Kapitel enthaltenen Bilder und sonstiges Drittmaterial unterliegen ebenfalls der genannten Creative Commons Lizenz, sofern sich aus der Abbildungslegende nichts anderes ergibt. Sofern das betreffende Material nicht unter der genannten Creative Commons Lizenz steht und die betreffende Handlung nicht nach gesetzlichen Vorschriften erlaubt ist, ist für die oben aufgeführten Weiterverwendungen des Materials die Einwilligung des jeweiligen Rechteinhabers einzuholen.

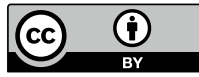

